\title{
The effect of the cell shape on compressive mechanical behavior of 3D printed extruded cross-sections
}

\author{
Sajjad Raeisi, Andres Tovar
}

\begin{abstract}
Additive manufacturing has been a promising technique for producing sophisticated porous structures. The pore's architecture and infill density percentage can be easily controlled through additive manufacturing methods. This paper reports on development of sandwich-shape extruded cross sections with various architecture. These lightweight structures were prepared by employing additive manufacturing technology. In this study, three types of cross-sections with the same 2-D porosity were generated using particular techniques. a) The regular cross section of hexagonal honeycomb, b) The heterogeneous pore distribution of closed cell aluminum foam cross section obtained from image processing and c) linearly patterned topology optimized 2-D unit cell under compressive loading condition. The optimized unit cell morphology is obtained by using popular two-dimensional topology optimization code know as 99-line code, and by having the same volume fraction as the heterogeneous foam. The upper edge of the unit cell was under distributed uniform loading and the lower edge was fixed. All the cross sections have the same cavity to wall area ratio on their 2-D configuration. The samples are extruded to produce 3-D CAD model of sandwich shape porous structures. The different samples are tested with universal compression machine and mechanical characteristics of the models are investigated. Furthermore, the energy absorption efficiency and load bearing capability of samples are studied. The results of the experimental procedure are compared to numerical simulations under quasi-static condition.
\end{abstract}

\section{Introduction}

For a long time, cellular structures were getting a sustained attention for their prominent applications in various industries such as automotive, aerospace, naval and biomedical. The most common application of cellular solids have focused on space-filling core materials (e.g., honeycombs and foam core sandwich panels), mainly to improve the flexural performance of the shell-based structures [1, 2]. Cellular material were rarely employed as main load bearing elements in engineering design. However the lightness and satisfactory compressive properties are the crucial necessities expected from the porous core inside the sandwich structures [3]. Mechanical properties of cellular solids are principally affected by the following basic features $[1,4,5]$ : a) relative density, b) base material properties, and c) pore architecture. Although, the impact of pore topology on mechanical behavior of cellular structures has not been widely investigated yet, the architecture of pores inside metallic foams was considered previously as the major characteristic affecting the deformation behavior of the foams. From this perspective, deformation behavior of them was classified into two main groups: bending-dominated and stretch-dominated deformation mechanism $[3,6]$. On the other hand, additive manufacturing techniques have opened revolutionary prospects for design and manufacturing of cellular solids with any arbitrary material configuration and sophisticated internal micro-architectures with sufficient accuracy [7]. Essentially, additive manufacturing provides us a strong tool to produce lightweight and functionally optimized cellular structures with extraordinary properties recently required in different industries.

Xiaolei Zhu et al. [5] developed a new modeling approach to simulate the closed-cell aluminum foam structures in 2-D. At first, X-ray computed tomography scanning images captured from real aluminum foam morphology were imported into MATLAB software. Then, the two-step mesh method was employed to discrete network by choosing proper number of nodes on the images. Finally, some FE models of aluminum foam material were generated directly via Abaqus software. The compression performance of aluminum foam, with various porosity were studied based on this technique. They validated their technique with numerical methods to prove the applicability of new modeling approach. F. Scarpa et al.[8] studied on compressive strength of hexagonal chiral in result of elastic buckling of the unit cells under quasi-static compressive loading condition. Analytical prediction, FE simulation and experimental test were used to study the mechanical behavior of honeycomb structures. A new type of chiral hexagonal honeycomb configurations with negative Poisson's ratio was introduced which had higher strength than axisymmetric ones under compressive loading.

Moreover, previous studies substantiated that loading direction [9, 10] and architecture [11] of additively manufactured structures have an essential role in collapse patterns and deformation mechanisms by using experimental tests and numerical methods. In Ref [12] an endto-end design process for topologically optimized cellular structures with minimized compliance was established. Homogenization was accomplished based on uniform distribution of the cellular structure to compute the macroscale structure for numerical model. Also they developed a new technique for generating the STL representation of the optimized part that is suitable for printing on typical industrial machines. Recently, an inclusive investigation [13] derived analytical predictions for mechanical properties (i.e. elastic modulus, Poisson's ratio, yield stress) of octagonal honeycomb structures according to the Euler-Bernoulli and Timoshenko beam theories. The elastic properties of the octagonal honeycomb was compared to those of honeycomb structures with different unit cell shapes such as: square, triangular, hexagonal, mixed, diamond, and Kagome. It was shown that the yield stress and elastic modulus of octagonal honeycomb were pretty similar to hexagonal one and lower than the other honeycombs with various unit cell.

This is the author's manuscript of the article published in final edited form as: 
In this paper, a series of samples with various pore architectures were studied using compression tests. We will investigate the deformation mechanism and compressive behavior of present porous structures according to the experimental results and the numerical simulations. In addition, we describe the relation between the microstructure and the load bearing capability of additively manufactured cross-sections. The characteristics of each model will be discussed based on deformation mechanism in micro level and local failures in structures.

\section{Materials and methods}

\section{A- Geometrical Modeling}

\section{A-1) Heterogeneous cross-section of a closed-cell Aluminum foam}

The cell morphology and microstructure of aluminum foams can be analyzed by optical observations [14] at different range of magnifications. The visual analysis is nondestructive, however, samples preparation usually needs cutting and polishing of the materials. Cell. Typically, a careful preparation of the materials is required to make sure that membranes and the interior of the cells appear in different brightness or color. As it is shown in Figure 1, an innovative approach of two dimensional modeling has been developed to acquire the CAD model of sophisticated geometries. In order to extract precise information from the images, aluminum foam sections need to be pre-processed before capturing digital photo. However, the pre-processing for this modeling technique is easy and remarkably economical, especially in comparison with micro X-ray computed tomography scanning used previously [5]. In this method, a black color sprayed on cellular structure and the cross section was wiped immediately with a soft cloth.

An ordinary digital camera was used to capture images from prepared section. The diagram of Figure 1 clearly illustrates the process of modeling. To begin, the images were imported into MATLAB software. Following this, matrix of Red-Green-Blue (RGB) colors combination was extracted from the image. Bases on the colors in each pixel, the matrix has values in range of $0-255$. Next, the enhancement process including removing background and increasing contrast were done. Then, the color value of were clustered to obtain binary image and some improvement like noise reduction of images were carried out. As it is represented in Figure 2.b, the black color in binary image represents the membrane walls in the closed-cell foam, while the white is corresponding to the cavities. In addition, the 52 detected holes on the heterogeneous cross-section are shown vividly in Figure 2.c with different colors.

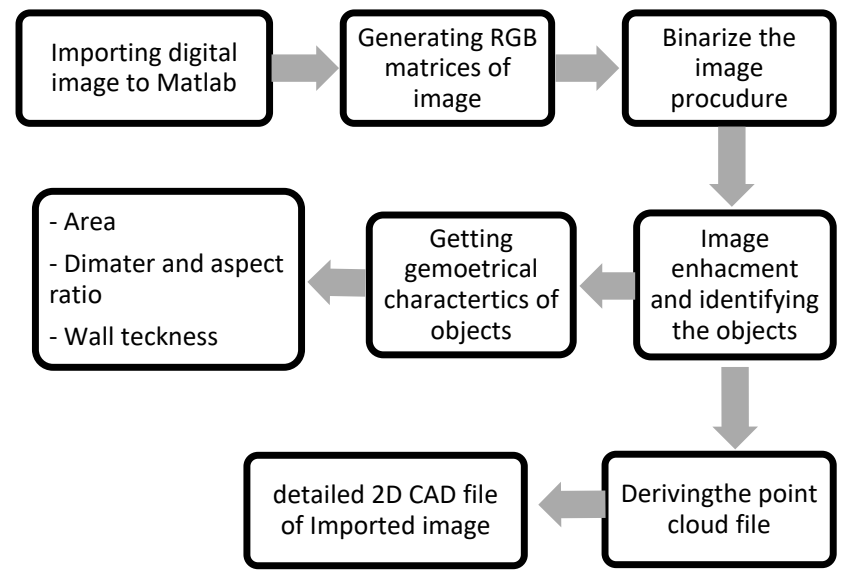

Figure 1: CAD process and getting geometrical characteristics based on MATLAB image processing

All the geometrical characteristics of each hole including equivalent diameter in perpendicular and horizontal direction, area based on the based on the number of pixels and their size corresponding to the digital image and thickness distribution of cell walls were extracted via MATLAB coding. Furthermore, the sketches obtained from point cloud were utilized to generate the 3-D CAD model (shown in Figure 2.d) by means of extruding the plane geometry in third direction.

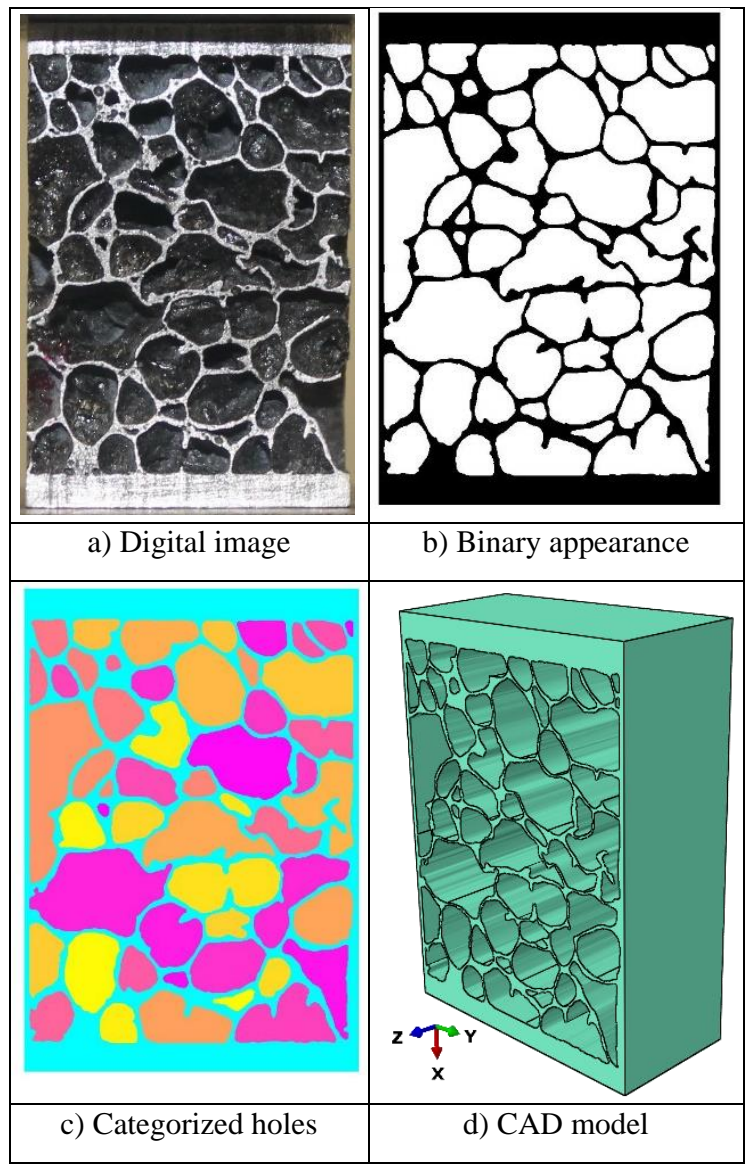

Figure 2: Steps related to CAD modeling process 
The heterogeneous shape was chosen In order to make comparison between the mechanical performance of a honeycomb with regular architecture and heterogeneous structure of closed-cell foam. The area of the hexagonal was designed to be equal to the mean area of cavities $\left(7.95 \mathrm{~mm}^{2}\right)$ on the foam cross-section. Furthermore, the thickness of hexagonal edge (almost $0.32 \mathrm{~mm}$ ) was same as the average thickness of the foam, which was calculated by image processing coding in Matlab and based on the process illustrated in Figure 1. The length of $1.75 \mathrm{~mm}$ was calculated to design a honeycomb shape equivalent to the heterogeneous model. The final sandwich model with honeycomb core is shown in Figure 3.

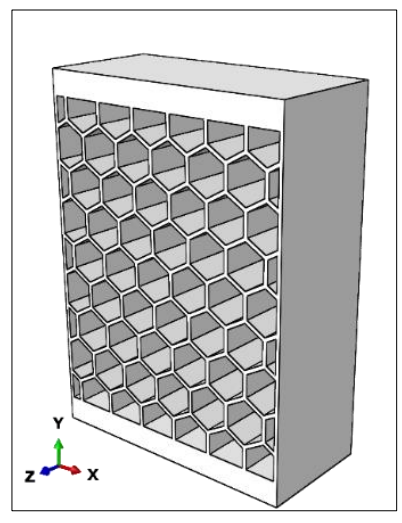

Figure 3: The final honeycomb model corresponding to the heterogeneous structure of the foam

\section{A-3) Topology optimized structure with the same volume fraction}

In pursuance of generating optimized cross section, popular Matlab topology optimization codes $[15,16]$ for compliance minimization of statically loaded structures was employed. By having the information obtained from process and outputs shown in Figure 1, the volume fraction of aluminum closed-cell foam cross section was calculated to be almost $25 \%$. The volume fraction of the cross section was measured by dividing the total area (pixels) occupied by black color in porous phase into the total area, which is the summation of the black and white color pixels in porous region, excluding the upper and lower plate of sandwich. For the sake of obtaining optimized unit cell, a rectangular design domain with the volume fraction of 25 were defined as 99 line Matlab code. As it is shown in Figure 4.a, the boundary condition of the fixed bottom edge and uniformly distributed loading condition on the upper edge were chosen to obtain the structure with minimized compliance. Figure 4.b represents the optimized unit cell with mentioned volume fraction equal to the both the previous model. Finally, the new sandwich structure was designed (shown in Figure 4.c) with the same dimensions as the foam core sandwich.

\section{B- Manufacturing and Investigation procedure}

In order to manufacturing the designed sandwich structures, the prepared STL files were imported into uPrint SE 3-D printer machine, working based on fused deposition modeling (FDM) Technology. In FDM technology the part are built layer-by-layer from the bottom up by heating and extruding thermoplastic filament. Pre-processing: a 3D CAD model is sliced via the connected software and calculates a path to extrude thermoplastic and any necessary support material are calculated.

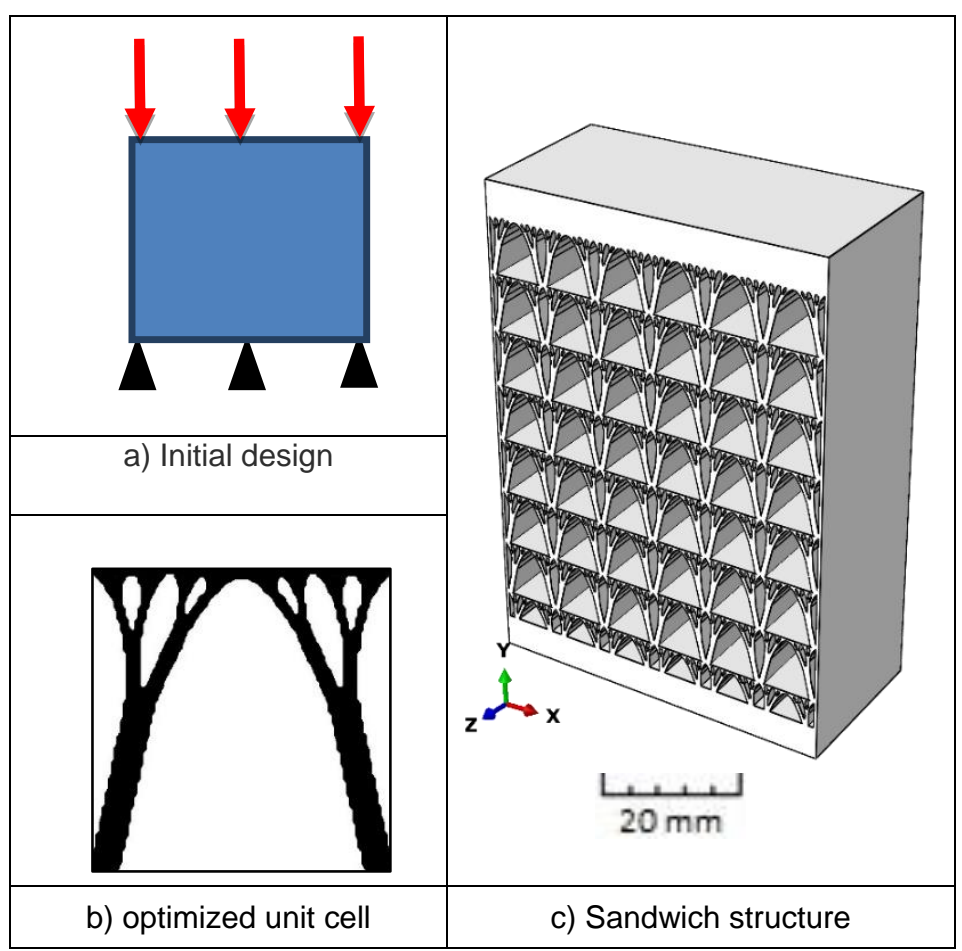

Figure 4: The design of topologically optimized structure

The process of FDM printing can be simply express as following steps [17]:

1. Production: thermoplastic will be heated up to a semi-liquid state by machine and pretty fine droplets will be deposited along the extrusion path established in the previous step. If the support or buffering is required to prevent unwanted deformation in semi-melted items, the machine deposits a removable material that acts as support structure.

2. Post-processing: after ending the printing process, a user removes support materials or dissolves them inside detergent and water. After drying the part, it will be ready for its application.

The models were sliced to layers of $10 \mu \mathrm{m}$ thickness. ABS plus material was used to print the models. The mechanical properties [17] of the material are given in Table 1.

Table 1: Mechanical Properties of ABS plus material

\begin{tabular}{|l|c|}
\hline Tensile Modulus & $220[\mathrm{MPa}]$ \\
\hline Poisson ratio & 0.35 \\
\hline Tensile Strength, Yield & $31[\mathrm{MPa}]$ \\
\hline Tensile Strength, Ultimate & $33[\mathrm{MPa}]$ \\
\hline Elongation at Break & $6[\%]$ \\
\hline
\end{tabular}

Figure 5demonstrates the post-processed manufactured sandwich structures through FDM 3-D printing technology. All the printed samples had closely same weight and volume fraction in porous region. The dimension of the printed samples is $60 \times 40 \times 20 \mathrm{~mm}$. The interior of the samples were filled with high density filling pattern and the models were printed along the same axis as the extrude line of cross section to ensure minimizing the demand on support structures. 


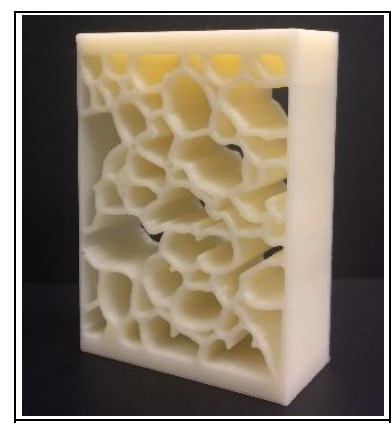

a) Foam based sandwich

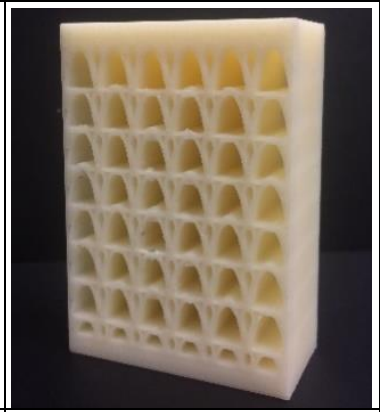

b) Optimized sandwich

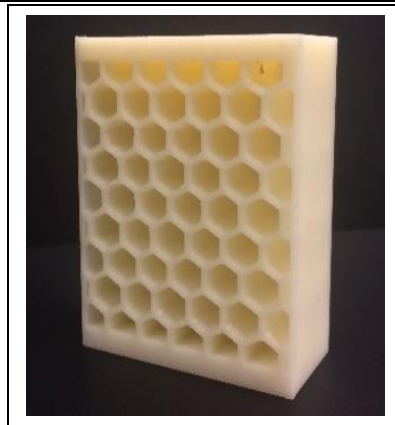

c) Honeycomb sandwich

Figure 5: Final printed structures

The numerical simulations were carried out using commercial FEA software LS-Dyna. In the simulations, a frictionless general contact algorithm was applied to prevent interpenetration during the deformation of the structures. It has to be noted that the elements type of the 8-node linear brick, reduced integration, hourglass control was picked up to mesh the models. Additionally, mesh refinement studies were done and element size varied in the range of 300-500 micron with considering to the accuracy requirements. In this study, the element elimination was not applied, however, by deleting failed elements more realistic results are expected. In the uniaxial compression, one end of the models was supported by a fixed rigid base and the other one was under a uniaxial compressive displacement. Furthermore, the manufactured samples shown in Figure 5 were considered to experimentally study the mechanical behavior of design structure. Firstly, a preload up to 20 Newton were applied on the samples while the gap between the jaws and the models was vanished completely with aim of abstaining accurate results and prevent the initial errors in experimental procedure. Experimental tests for reinforced and base specimens have been performed under a quasi-static condition with a strain rate of $1.5 \times 10^{-3}$.

\section{Results and Discussion}

The obtained stress-strain curve for the various samples are presented in Figure 6. For the foam-based model, the plot include three regions. Firstly, the structure deformed in elastic zone with no damage or failure. However, after the strain of $2 \%$, a local yield in internal microstructure resulted in constant stress values. The left edge of the sample shown in Figure 8.b can be considered as this early yield. By increasing the strain, the others walls underwent the deformation. As it's shown in Figure 8.c, the edge located in the right side of the structure was experiencing deformation in this stage. Also, the graph of stress in Figure 6, reveal that the stress values after 2.5\% is increasing up to $3.7 \%$. This hardening stage is corresponding to the bending of the second edge. As the numerical simulation proves in the Figure 8, the more strain resulted in remarked decline in stress value due to the bending in the right edge. From then on the stress Page 4 of 7 reached a plateau region by the end of the compression. Although, the strength of the foam based structure was significantly lower than the other ones, the material did not experienced major failure or drop in stress value. It can be predicted that foam-based model has ability to apply pretty high values of stress. In addition, this structure were failed only at special point and rest of the internal wall has remained almost unchanged. Moreover, Figure 7 vividly shows the limited number of local failures in the foam based structure and considerable amount of undamaged walls inside the sandwich. Similarly, the limited of unaffected regions can be recognized by blue color (representing a low level of Von-Misses stress) in FE analysis. The most important reason of comparably lower stress in this model is the limited number of cells and walls carry the load simultaneously.

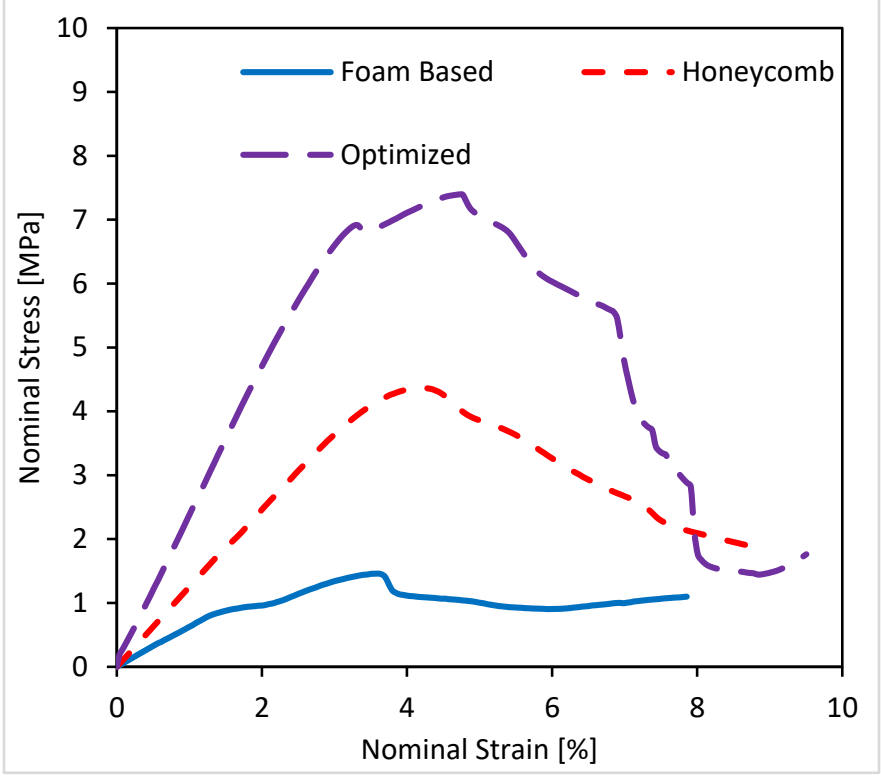

Figure 6: Experimental stress-strain curves of different samples

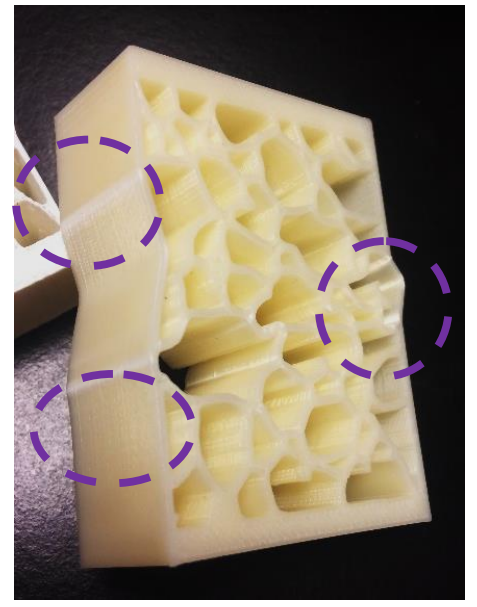

Figure 7: Limited number of local failures in the foam based structure

The elastic behavior of the regular honeycomb model denoted the stiffer structures compare to heterogeneous one. As Figure 6 represents, the nominal stress on the honeycomb model increased steadily until the strain of $4 \%$. The sections of $b$ and $c$ in Figure 10, illustrated the consistent behavior of the honeycomb structure up to that point. The major difference between honeycomb and the foam model is the number of cells experienced the deformation at the same time. As Figure 10.b demonstrates, all most all the hexagonal cell were deformed at the very initial stage of compression. 


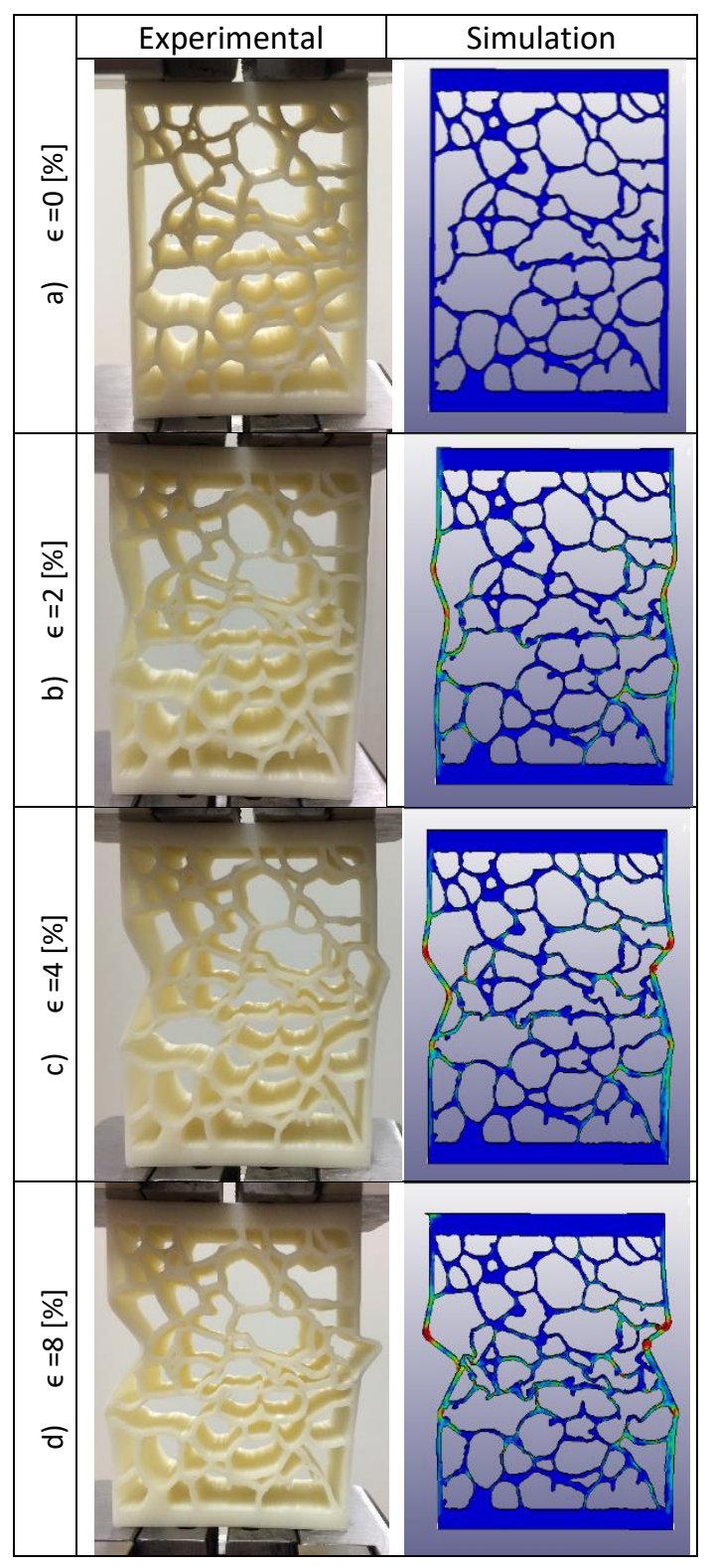

Figure 8: Deformation in the foam-based model

Likewise, Figure 9 obviously shows the yield regions were appeared in approximately all the cell in contrast with the limited number of failures in the foam based model. The decline in stress value at the strain of $4 \%$ is due to initiation of the deformation band (Figure 10.c) and the stress graph in Figure 6 was dropped continually after this point. The steady decrease in energy absorption of the structures is because of development of deformation where it finalized at the higher compression strain shown in Figure 10.d.

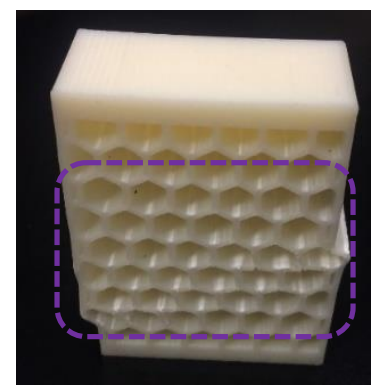

Figure 9: Simultaneous yield on the different cell walls

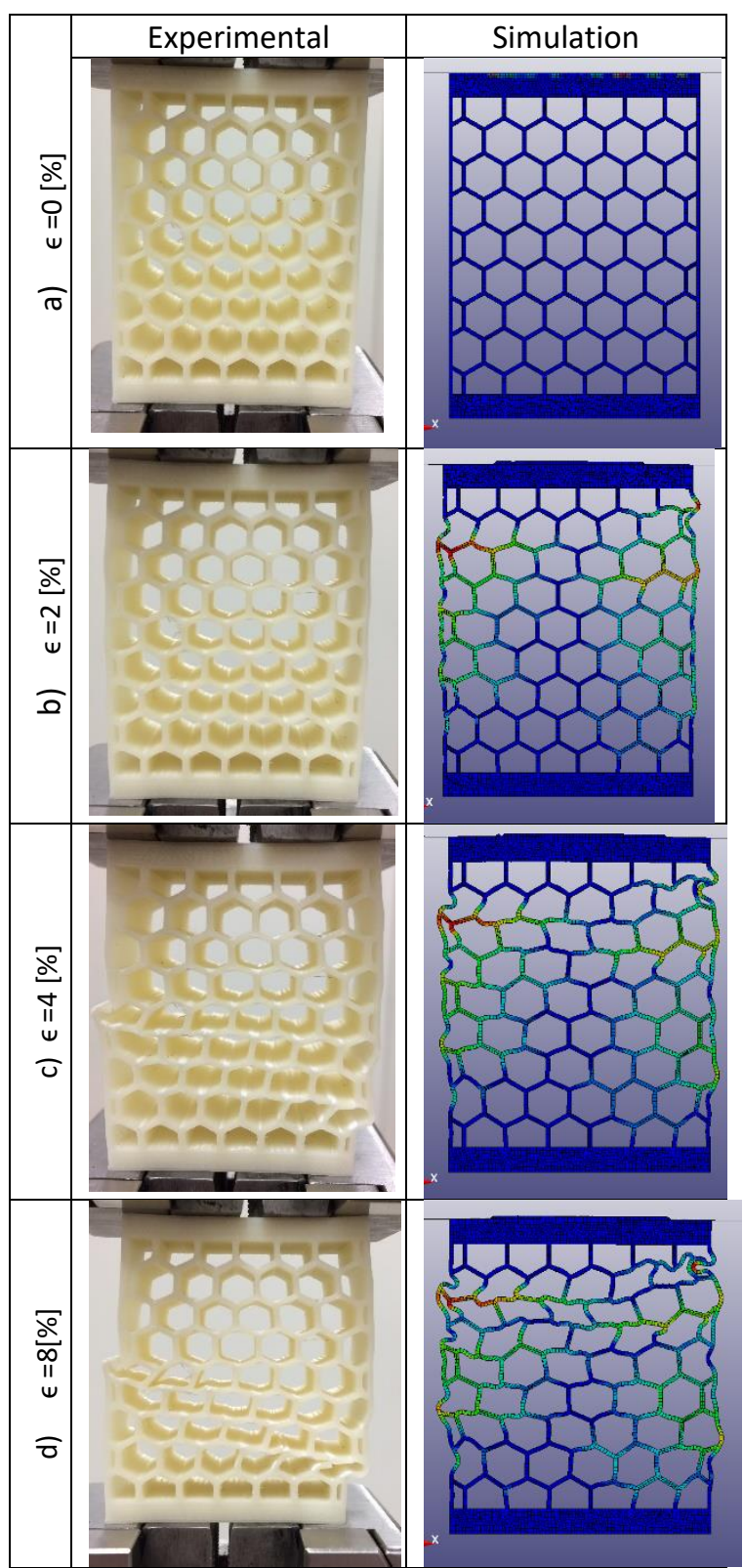

Figure 10: Deformation in the Honeycomb model

The optimized sample, had the highest Young modulus and Figure 6 represent the sharp increase in stress value at elastic region. Although local deformations occurred at lower strain values for both the experimental and numerical examinations shown in Figure 11b, the optimized sandwich structure continue elastic behavior until the strain of $3.3 \%$ due to homogeneity and nature of the unit cell which is topologically optimized for load bearing purpose. Similar to most of the cellular structures, deformation bands emerged by growing the compressive strain. The bands developed with the angle between 20 and 35 degree [18] which ensuing the sliding of porous layer on one another shown in Figure 11.d. The shear stress due to sliding layer decreased the reaction force of the deformed structure appeared as softening region in Figure 6 .

The two significant properties of the sandwich panel are shown in Table 2. As it is discussed before, stiffness of the foam based material is the minimum value compared to the other structures. The Young modulus of the hexagonal structure is almost twice as the foam based one. This sharp increase in stiffness is due to regularity and continuity of the honeycomb structure. The optimized structure has 
the maximum value of elastic modulus to be roughly four times of the heterogeneous one. Besides homogeneity, the design of the unit cell which was based on the minimized compliance is the reason of huge growth in elastic properties of the latter model.

Table 2: Mechanical properties of the different models

\begin{tabular}{|l|c|c|}
\hline Model & Young Modulus [MPa] & Peak Stress [MPa] \\
\hline Foam Based & 62.7 & 1.45 \\
\hline Honeycomb & 124.1 & 4.37 \\
\hline Optimized & 234.4 & 7.39 \\
\hline
\end{tabular}

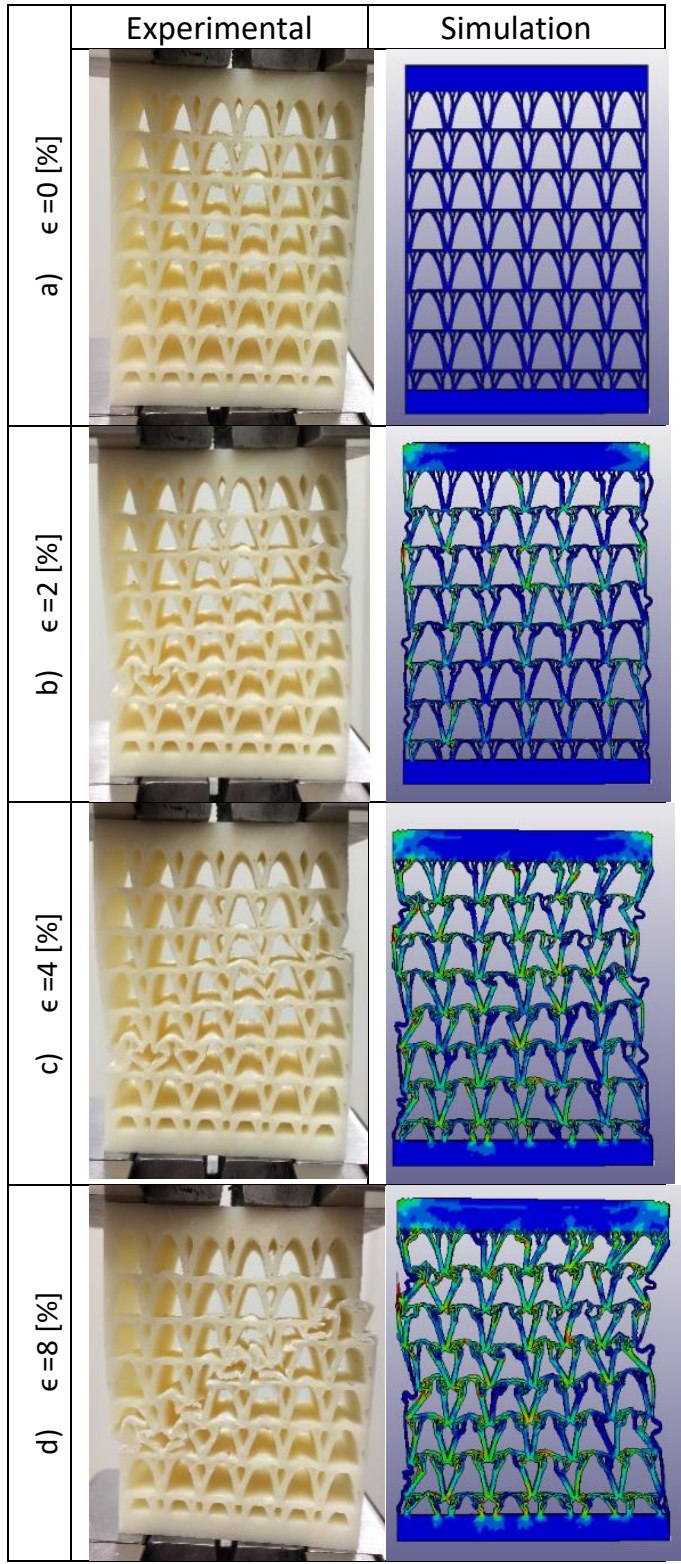

Figure 11: Deformation in the Honeycomb model

\section{Energy absorption efficiency}

Energy absorption efficiency (EAE) is a noteworthy feature of cellular structures, which represent the comparison between the strain energy absorbed by the real material and that of an ideal imaginary energy absorber [19]. Besides, by using this efficiency graph we can obtain some of the structure feature which are not available to get through stress-strain curve of the sum of porous materials. EAE curve Page 6 of 7 can be used for determining the yield strain of the structure here. EAE curve undergoes substantial changes throughout strain increment. The first relative maximum in the curve represents the material yielding [20]. The EAE parameter is calculated using the following equation:

$\eta=\frac{\int_{0}^{\varepsilon} \sigma d \varepsilon}{\sigma_{\max } \times \varepsilon}$

Where $\eta$ is energy absorption efficiency, $\sigma$ is stress, $\varepsilon$ is strain, and $\sigma_{\max }$ is maximal stress up to the strain of $\varepsilon$.

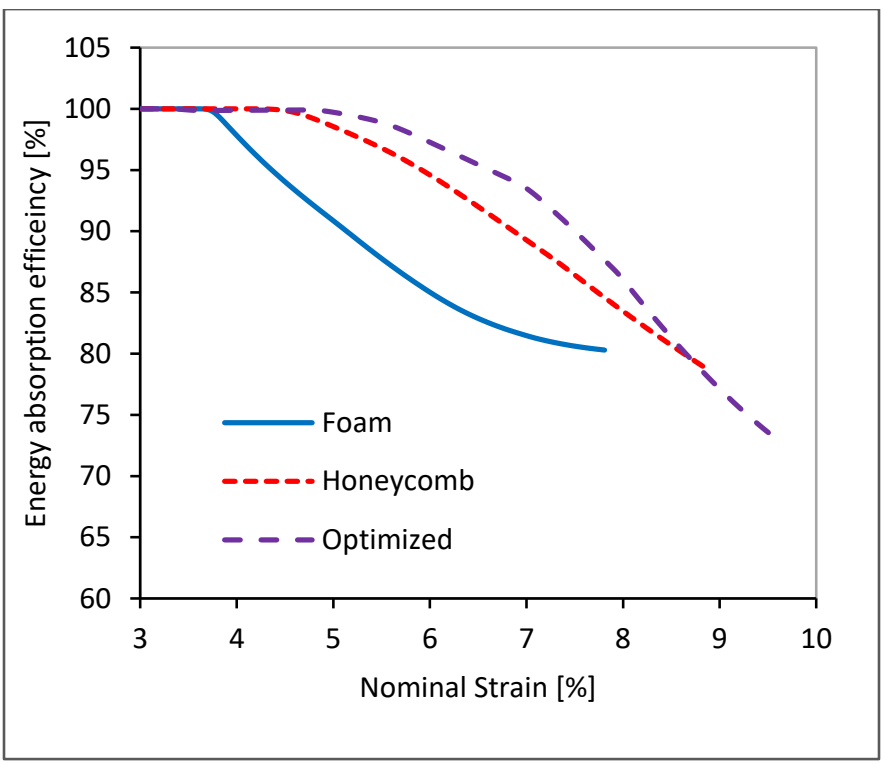

Figure 12: Energy absorption efficiency of the various structures

The EAE graph of the different samples is reported in Figure 12. The foam-based sandwich was perfectly efficient up to almost $3.8 \%$ of strain. It mean that the material performed completely elastic in this range with continuous increase in stress value by growth in strain. The local failures which resulted in plastic hinges at some regions cause a softening regime in stress curve, followed by decreasing the energy absorption efficacy. The graph obviously shows that the efficiency of this structure was reduced significantly at early strains. However, by increasing the strain, more walls will undergo the deformation and EAE inclined to remain stable for rest of compression process, or at least prevent sharp declines. The honeycomb sandwich behave like an elastic material until almost 5\% of strain. Afterward, efficiency of the structure gradually falls. The optimized model has remained almost elastic for higher values of strain compare to previous one. Nonetheless, a gradual drop in efficiency is followed by remarked drop due to formation of deformation bands inside the structure. The peak stresses reported in Table 2 are affected by both the elastic modulus and yield strain. For instance, topologically optimized structure had the highest amount of peak stress because of comparably large elastic modulus beside higher yield strain based on the graphs.

\section{Conclusion}

In this study, three types of cellular structure were used to produce sandwich structure. The first one had a perfectly heterogeneous structure inspired by cross section of a closed-cell aluminum foam. The foam architecture was generated through an innovative cheap technique for 2-D modeling, based on image processing. The next structure was a regular hexagonal honeycomb with dimensional 
features such as holes area and wall thickness same as the foam. Finally, a promising topology optimization code was used to generate a unit cell with minimum compliance under compressive loading. The optimized unit cell was used to design a sandwich structure with repetitive cellular core. All the samples had same volume fraction and similar dimensions to study the effect of cell morphology on mechanical behavior of sandwich panels. The results shows that, foam-based model had the lowest stiffness, as well as the less area damaged under compressive loading. The honeycomb and the optimized structures had higher capability in load bearing, beside significant damages under comparably small compressive strain.

\section{References}

1. Gibson, L.J. and M.F. Ashby, CellularSolids: Structureand properties, second edition. 1999, Cambridge: Cambridge university press.

2. M.F. Ashby, A.G.E., N.A. Fleck, L.J. Gibson, J.W. Hutchinson and H.N.G. Wadley, Metal Foams: A Design Guide. 2000, Burlington: Butterworth-Heinemann.

3. Alkhader, M. and M. Vural, Mechanical response of cellular solids: Role of cellular topology and microstructural irregularity. International Journal of Engineering Science, 2008. 46(10): p. 1035-1051.

4. $\quad$ Ashby, M.F., The properties of foams and lattices. Philosophical Transactions of the Royal Society A: Mathematical, Physical and Engineering Sciences, 2006. 364(1838): p. 15-30.

5. Zhu, X., et al., A novel modeling approach of aluminum foam based on MATLAB image processing. Computational Materials Science, 2014. 82: p. 451-456.

6. Guest, S.D. and J.W. Hutchinson, On the determinacy of repetitive structures. Journal of the Mechanics and Physics of Solids, 2003. 51(3): p. 383-391.

7. Gibson, I., D.W. Rosen, and B. Stucker, Additive Manufacturing Technologies: Rapid Prototyping to Direct Digital Manufacturing. 2010: Springer US.

8. Scarpa, F., et al., Elastic buckling of hexagonal chiral cell honeycombs. Composites Part A: Applied Science and Manufacturing, 2007. 38(2): p. 280-289.

9. Amirkhani, S., R. Bagheri, and A. Zehtab Yazdi, Effect of pore geometry and loading direction on deformation mechanism of rapid prototyped scaffolds. Acta Materialia, 2012. 60(6-7): p. 2778-2789.

10. Amirkhani, S., R. Bagheri, and A. Zehtab Yazdi, Manipulating failure mechanism of rapid prototyped scaffolds by changing nodal connectivity and geometry of the pores. Journal of Biomechanics, 2012. 45(16): p. 28662875.

11. Kadkhodapour, J., H. Montazerian, and S. Raeisi, Investigating internal architecture effect in plastic deformation and failure for TPMS-based scaffolds using simulation methods and experimental procedure. Materials Science and Engineering: C, 2014. 43: p. 587-597.

12. Robbins, J., et al., An efficient and scalable approach for generating topologically optimized cellular structures for additive manufacturing. Additive Manufacturing, 2016.

13. Hedayati, R., et al., Mechanical properties of additively manufactured octagonal honeycombs. Materials Science and Engineering: C, 2016. 69: p. 1307-1317.

14. Banhart, J., Manufacture, characterisation and application of cellular metals and metal foams. Progress in Materials Science, 2001. 46(6): p. 559-632.

15. Sigmund, O., A 99 line topology optimization code written in Matlab. Structural and Multidisciplinary Optimization, 2001. 21(2): p. 120-127.

16. Liu, K. and A. Tovar, An efficient $3 D$ topology optimization code written in Matlab. Structural and Multidisciplinary Optimization, 2014. 50(6): p. 1175-1196.

17. Stratasys. uPrint SE. 2012; Available from: http://www.stratasys.com/3d-printers/idea-series/uprint-se.

18. Kadkhodapour, J. and S. Raeisi, Micro-macro investigation of deformation and failure in closed-cell aluminum foams. Computational Materials Science, 2014. 83: p. 137-148.

19. Baumeister, J., J. Banhart, and M. Weber, Aluminium foams for transport industry. Materials \& Design, 1997. 18(4-6): p. 217-220.

20. R. Florek, F.S., M. Nosko, J. Harnúšková, Compression test evaluation method for Aluminum foam parts of different alloys and densities. Powder Metallurgy Progress, 2010. 10(4).

\section{Contact Information}

Andres Tovar

Indiana University-Purdue University Indianapolis

723 W. Michigan St., SL 260N

Indianapolis, IN 46202-5132

Phone: (317) 278-7090

Fax: (317) 274-9744

Email: tovara@iupui.edu 